\title{
Scheduling and Flexible Manufacturing
}

\author{
Peter Brucker, Universität Osnabrück
}

With new production technologies special scheduling problems have been formulated. In this survey we will discuss some specific features of scheduling in flexible manufacturing systems like general shop scheduling problems, scheduling for multi-purpose machines, combined scheduling and transportation problems, families of jobs (operations) and changeover costs, batching, and due-date scheduling.

The general shop problem is a generalization of the open-shop problem and the job-shop problem. Each job consists of a set of operations. Associated with the operations there are processing times and machines on which each operation must be processed. Between operations precedence constraints are given and we have to find a feasible schedule which minimizes some objective function.

A multi-purpose machine is a machine which is equipped with different tools. An operations can be processed on all machines equipped with the tool needed for doing the operation. The multi-purpose machine scheduling problem is to find a feasible assignment of operations to machines and to schedule the operations on the assigned machines. Special general shop problems with multi-purpose machines are discussed.

As a combined scheduling and transportation problem a general shop problem is considered in which jobs are to be transported from machine to machine. This transport is done by one or more robots and takes time. Cases with (i) only one robot, (ii) a few robots, (iii) a sufficient large number of robots are considered. Problems with families of operations and changeover times if two succeeding operations on the same machine belong to different families are equivalent to corresponding combined scheduling and transportation problems. Another problem is the batching problem. Here the jobs to be processed on the same machine must be grouped together into batches. All jobs in the same batch are processed jointly and their finish time is the finish time of the last job in the batch (batch availability). If a batch goes onto a machine there is a set-up time. The problem is to find a partioning of all jobs into batches and a schedule for these batches such that some objective function like mean flow time is minimized.

Finally scheduling problems involving due-dates which arize in connection with just-in-time production strategies are discussed. 